\title{
Habilidad de análisis fonético y adquisición de la lectura en los sistemas alfabéticos
}

\section{María Clemente*}

Universidad de Salamanca

\section{INTRODUCCION}

A la vista de un importante número de trabajos ${ }^{1}$ podemos decir que la lectura en los sistemas alfabéticos implica la utilización de dos vías de acceso al léxico. Una vía es la fonética, en la que el lector establece correspondencias entre grafemas y fonemas ${ }^{2}$, esto es, aplica reglas de representación fonológica. La otra vía es la global o directa, en la cual la palabra se capta como una totalidad, algo semejante a como se identifica un número.

Asumiendo el punto de vista de las dos vías, voy a dedicar las siguientes. páginas a plantear un aspecto peculiar de la utilización de la vía fonética: la babilidad de análisis fonético y su relación con la adquisición de la lectura en los sistemas alfabéticos. Mi reflexión va a tener por el momento un carácter más psicológico que pedagógico, más centrado en los procesos que intervienen al leer que en técnicas concretas de enseñanza. Conocer los procesos básicos que tienen lugar en el aprendizaje de la lectura es condición indispensable para que cada profesor pueda elegir o desarrollar métodos, técnicas y procedimientos adecuados que faciliten al niño la adquisición de la capacidad de leer ${ }^{3}$.

Pero debo justificar por qué, asumiendo la incidencia de las dos vías, voy a ocuparme aquí sólo de la fonética. Creo que en los últimos años ésta ha tenido cierta «mala prensa», quizá por varios motivos: por una parte, los defensores de los llamados métodos fónicos o sintéticos consideran esta vía como única, despreciando la palabra como unidad de significado y proponiendo una práctica poco significativa y desde luego nada motivadora; por otra parte, la importancia del globalismo como principio general en pedagogía infantil ha llevado a los defensores de los métodos globales a argumentar que la palabra y en su caso la frase son las unidades básicas sobre las que debe centrarse la adquisición de la lectura, considerando de este modo la vía directa como la única relevante. Es preciso reconocer que el lector hábil, el buen lector, puede que utilice predominantemente la vía global, pero no de forma exclusiva. Basta reflexionar sobre una propia experiencia como lectores para darse cuenta de que la vía fonética se utiliza continuamente junto a la directa; por ejemplo, cuando-encontramos palabras que nunca habíamos visto escritas e incluso cuyo significado desconocíamos pero que somos capaces de leer (aunque luego debamos mirar el diccionario), también la utilizamos para distinguir palabras casi idénticas visual y fonéticamente (casa/caso, sueño/dueño, cal/sal, muestra/nues-

* Dirección de la autora: Universidad de Salamanca. Departamento de Metodología Educativa. Paseo Canalejas, 168. Salamanca. 
tra, etc.). El interesante estudio de Rubenstein, H., Lewis, S. S. y Rubenstein, M. A. (1971) muestra claramente cómo lectores adultos, en este caso universitarios, siguen utilizando la vía fonética o indirecta ${ }^{4}$.

Junto a estas consideraciones hay un hecho que parece olvidarse con frecuencia: me refiero a las peculiaridades del sistema de escritura alfabético, sistema cuyas unidades mínimas son las letras (grafemas) que representan fonemas y no palabras. Ello implica que el conocimiento de esas unidades mínimas es imprescindible para leer en un sistema alfabético. Este hecho pone de manifiesto dos cuestiones claras: primero, que es probable que los procesos para aprender a leer (e incluso para leer) no sean del todo idénticos en los distintos sistemas de escritura (logográfico, silábico y alfabético); segundo, que quizá la capacidad de análisis fonétiço sea una habilidad totalmente ligada a la lectura en el sistema alfabético. Por ello, antes de tratar esta segunda cuestión, que es el motivo de este trabajo, presentaré un breve resumen del desarrollo histórico de los sistemas de escritura, a fin de aclarar la evolución que han seguido las formas de representación del lenguaje oral y observar las particularidades de los diferentes tipos de escritura.

\section{Sistemas de escritura}

La tesis mantenida por Gelb, enormemente coherente y fundamentada, es a grandes rasgos la siguiente. La evolución de la escritura va inevitablemente de las representaciones logográficas a las silábicas y después a las alfabéticas, con unas etapas previas que ẹl llama etapa de imágenes y. etapa semasiográfica (Cuadro I).

CUADRO I

Etapas de la escritura según Gelb.

1. Antes de la escritura: imágenes.

2. Precursores de la escritura: semasiografía.

- Procedimiento de descripción-representación.

- Procedimiento de identificación-memorización.

3. Escritura propiamente dicha

- Logosilábica: sumeria

- Silábica: elamita semítica

$\begin{array}{ll}\text { elamita } & \text { semítica } \\ \text { hurita } & \text { occidental }\end{array}$

hitita china

$\begin{array}{cc}. & \text { hurita } \\ & \text { occiden } \\ \text { (fenicia } \\ \text { hebrea }\end{array}$

- Alfabética:

aramea)

griega

aramea (vocalizada)

hebrea (vocalizada)

latina

lenguas de India

Según este autor, en un principio, cuando el hombre quería representar algo, utilizaba dos procedimientos. Uno consistía en la descripción-representación, parecido a la manifestación estética, pero en el que sólo importaba la comunicación y expresión. El otro era la identificación-memòrización, en el que cierto símbolo servía para la identificación o registro de tal persona u objeto.

La escritura nacería de esta segunda forma, puesto que en ella se pretende más ayudar a recordar e identificar el acontecimiento u objeto que a describirlo. Así se van haciendo convencionales algunos elementos representados, y como éstos tienen un nombre en la lengua hablada, se hace pronto la correspondencia. A partir de aquí, cuando se descubre que las 
palabras pueden ser representadas por algún signo, nace un nuevo método de comunicación humana. Tal procedimiento conduce a un sistema completo de signos de palabras o logogramas, en el que cada signo representa la palabra de forma global. Este sistema se opondrá al de descripción-representación artística donde nada es convencional.

Sin embargo, un sistema logográfico puro es realmente complejo si tenemos en cuenta que cada palabra, incluso a veces cada frase o expresión, es un signo diferente. Esto dificulta enormemente el reconocimiento, por parte del lector, de todas las palabras escritas. Un dato actual puede ser ilustrativo: la escritura china, predominantemente logográfica, tiene al menos unos cincuenta mil signos, de los que un letrado adquiere a lo largo de su vida probablemente unos cuatro mil, y recurriendo constantemente al diccionario. De hecho, Gelb piensa que un sistema totalmente logográfico no puede subsistir por antieconómico y constata cómo el chino, considerado el sistema logográfico más puro, ha llegado a incorporar signos silábicos.

Así, pues, para que un sistema se convierta en eficaz es preciso simplificarlo dándole al signo un valor fonético. Gelb considera la fonetización como la etapa más importante de la historia de la escritura. La primera fonetización da origen a los sistemas silábicos, de los cuales destacan cuatro: cuneiforme-elemita, semítico-occidental, chipriota y japonés, que se caracterizan por una fónetización en la secuencia silábica, lo cual supone. ya un ahorro muy considerables. Aun así, el sistema evolucionó hasta llegar al alfabeto.

El primer alfabeto es el griego, procedente del silabario semítico occidental. En él la demarcación o elaboración de las vocales deja en un valor simple a los signos consonánticos.

Los alfabetos principales son de tres tipos:

Tipo I. Griego-latín. En él las vocales están indicadas por signos distintos (ta, te, ti, to, tu).

Tipo II. Arameo, hebreo, árabe. En él las vocales están indicadas por puntos diacríticos distintos $\left(t, t, t, t, t^{\circ}\right)$.

Tipo III. Indio, etíope. En él las vocales están indicadas por puntos diacríticos sujetos al signo o por modificación de éste.

La característica fundamental del alfabeto radica en la enorme disminución de signos respecto de su antecesor, el silabario, ya que al representar los sonidos consonánticos y vocálicos de manera autónoma, los reduce a una treintena. Así se convierte en el sistema más económico, puesto que conociendo este breve conjunto de signos (las letras del alfabeto) somos capaces de leer todas las palabras de nuestra lengua. Sin embargo, esta simplificación tiene una contrapartida, que se deriva del hecho de que los signos representan fonemas que carecen en sí mismos de significado. Lo cual supone que las tareas que deben realizarse para aprender a leer en un alfabeto no se pueden reducir al reconocimiento global y visual de la palabra, sino que implican una serie de mecanismos más complejos (de los que hablàré en el siguiente apartado).

\section{Habilidad de análisis fonéticos y adquisición de la lectura}

Considero correcta la idea de A. Content (1984), según la cual, el sistema alfabético se basa en una representación fonológica de la lengua ha- 
blada, dado que las letras representan los sonidos de una lengua. Ello implica que quien aprende a leer en este sistema va a tener que realizar tareas que le lleven a hacer explícitas reglas de correspondencia grafemafonema. Estas tareas consisten en la manipulación e identificación de fonemas, la segmentación de las palabras en fonos, el aislamiento de fonos en la corriente acústica, etc. En definitiva, se trata de una auténtica actividad metalingüística que podemos denominar Análisis fonético de la palabra, el cual no se requiere para leer en los sistemas logográficos ni, por supuesto, para comprendé y practicar la lengua oral. Esta idea está corroborada por un buen número de trabajos, que vienen a demostrar la existencia de una relación directa entre la capacidad de análisis fonético y el aprendizaje de la lectura en el sistema alfabético.

Los trabajos de Liberman (1973) y (1974) analizaron la relación entre el nivel lector de niños de segundo curso de enseñanza primaria y los resultados de un test de «recuento» realizado el año anterior con esos niños, y mostraron que el grupo de niños que constituía el nivel inferior en la clase de lectura había fracasado en dicho test, mientras que el grupo de buenos lectores había tenido éxito en él. Un estudio longitudinal realizado por Foz y Routh entre 1980 y 1983 muestra igualmente esta relación. La prueba en este caso consistía en descomponer frases en palabras, palabras en sílabas y éstas en fonos. Se aplicó a tres grupos de niños, según el nivel lector (buenos, medios y malos lectores) y se observó que mientras que los dos primerôs grupos obtenían buenos resultados en la descomposición de frases, palabras'y de sílabas en fonos, el tercer grupo alcanzaba un nivel aceptable en la identificación de palabras (un 80 por 100 de aciertos las niñas y un 41 por 100 los niños) y sílabas (un 77 por 100 y un 40 por 100 de aciertos, respectivamente), pero en el análisis de fonemas el resultado era sólo de un 5 por 100 y un 2 por 100 de respuestas correctass

Morais, Cluytens y Alegría (1984) compararon dos grupos de niños, uno de disléxicos y otro de lectores normales de primero y segundo nivel, y constataron que sólo los disléxicos tenían dificultades serias para realizar tareas de segmentación fonética, aunque mostraban capacidades similares a las del grupo de lectores normales en tareas de segmentación de sonidos no verbales. Por su parte, Bradley y Bryant (1978) dan cuenta también de la relación entre un buen nivel lector y la ausencia de errores en el análisis de la palabra, y, paralelamente, de cómo los malos lectores cometen un alto índice de fallos (un 85 por-100 aproximadamente) en este tipo de análisis. Estos mismos autores realizaron más tarde un estudio longitudinal, concluido en 1983 y semejante a los de Liberman, en el que se relacionan el buen nivel lector de niños de siete y ocho años con los resultados satisfactorios obtenidos por estos tres años antes en pruebas de categorización de sílabas con un fono común.

Esta tarea de análisis fonético de la palabra, que parece inevitable en la adquisición de la lectura en un sistema alfabético, no es, sin embargo, fácil para el hiño prelector. Y ello se debe sobre todo a que nos es imposible aislar todos los fonemas consonánticos en la corriente acústica (sobre todo, si se trata de consonantes explosivas y nasales), como muestran, por ejemplo, los trabajos llevados a cabo por Liberman y sus colaboradores en los laboratorios Haskins (y publicados en 1967). Esto lleva a pensar que es la sílaba y no el fonema la unidad aparente y reconocible en la corriente acústica. Lo cual coincide perfectamente con las observaciones de Ferrëiro y Teberosky (1979), quienes consideran que las intuiciones espontáneas del niño cuando se enfrenta al lenguaje escrito son hipótesis silábicas y no* fonéticas. 
Por todo ello, el problema básico en la enseñanza de la lectura (en el sistema alfabético) consiste en entender cuándo llega el niño a captar la existencia de esas entidades mínimas y abstractas que son los fonemas, cómo adquiere esa capacidad y cómo es posible ayudarle a realizar este tipo de análisis.

\section{Habilidad de análisis fonético y desarrollo}

La cuestión de cuándo llega el niño a adquirir la habilidad de analizar fonéticamente las palabras ha sido estudiada en diferentes ámbitos lingüísticos con conclusiones bastante aproximadas. Rosner y Simon (1971) plantearon a niños de cinco y seis años tareas de segmentación de palabras en sílabas y de sílabas en fonos, y los resultados mostraron que el 80 por 100 de los niños de estas edades llevaban a cabo correctamente la primera tarea, pero tan sólo el 20 por 100 realizaban la segunda. Liberman y otros (1974) compararon la capacidad de segmentación de la palabra en grupos de segundo y tercero de parvulario y primer curso de primaria. Pedían al niño dar tantos golpes como segmentos tuviera la palabra, unas veces atendiendo a las sílabas y otras a los fonos, todo ello con ejemplos oportunos. Los resultados de los porcentajes de niños que realizaban los dos tipos de análisis (silábico y fonético) los mostramos en el Cuadro II.

\section{CUADRO II}

Análisis fonético y silábico (Liberman y otros, 1974)

\begin{tabular}{lccc}
\hline & $20^{\circ}$ párvulos & $3 .{ }^{9}$ párvulos & $1 .^{9}$ primaria \\
\hline Fonos & 0 & 17 & 70 \\
Silabas & 45 & 48 & 90 \\
\hline
\end{tabular}

Parece claro que la habilidad de análisis fonético aparecía en el tercer curso de párvulos (seis años) y se dominaba mejor en el curso siguiente, mientras que la capacidad de reconocer sílabas se manifestaba mucho antes. Por su parte, Alegría y Morais (1979) realizaron una prueba a niños de primero y segundo de primaria que consistía en añadir o sustraer cierto fono tanto en palabras como en pseudopalabras. Los porcentajes de respuestas correctas aumentaban considerablemente a lo largo del primer curso. En noviembre (de primero) los porcentajes de respuestas correctas eran de 16 por 100 para la supresión y 26 por 100 en adición. En febrero (también de primer curso) aumentaban a 34 por 100 y 64 por 100, respectivamente; finalmente, en noviembre de segundo curso, eran ya de 74 por 100 en supresión y 79 por 100 en adición.

Zhurova, en 1973, llevó a cabo una experiencia con niños de edades comprendidas entre cuatro y siete años, que consistía en aislar fonos iniciales en palabras. La tarea era resuelta en estos términos: entre cuatro y cinco años, el 12 por 100 de los niños; entre cinco y seis años, el 39 por 100 , y a los seis y siete años, todos los niños a los que se aplicó la prueba.

Se da, pues, la circunstancia de que esta habilidad de análisis fonético se manifiesta a los seis años aproximadamente. Pero debemos tener en cuenta que prácticamente todos los trabajos comentados se han realizado con niños escolarizados, lo cual nos lleva a cuestionarnos si dicha habilidad es producto de la simple maduración a una determinada edad o, por 
el contrario, es el fruto de las actividades escolares, incluidas las relacionadas con la lectura. Esta cuestión tiene, sin duda, importantes implicaciones pedagógicas.

En lo que sigue, voy a presentar algunos estudios que parecen arrojar un poco de luz sobre el problema.

\section{Adquisición de la capacidad de análisis fonético}

Alegría, Pignot y Morais (1979) han comparado la capacidad de segmentación, tanto silábica como fonética, en niños de primer año de primaria, atendiendo al método (fonético o global) con que aprendían a leer. Los resultados mostraron que sólo los niños que aprendían con un método fonético, y que, por tanto, realizaban habitualmente análisis fonético de forma explícita, tenían una buena preparación en segmentación fonética, mientras que está preparación era muy deficiente en los que aprendían con métodos globales. Según esto, parece ser que el aprendizaje es más importante para el dominio del análisis fonético que la simple maduración. Profundizando en esta hipótesis, Morais, Cary, Alegría y Bertelson (1979) realizaron un estudio semejante al anterior. En este caso se evaluaba la capacidad de segmentar y fusionar fonos de dos grupos de adultos, uno de analfabetos por causas sociales, y otro recientemente alfabetizado. La tạrea consistía en añadir o sustraer segmentos fonéticos al principio de palabras y pseudopalabras y los resultados, que se muestran en el Cuadro III, en forma de porcentajes de respuestas correctas de los dos grupos, nos indican claramente que los analfabetos fueron muy inferiores en todos los casos al grupo de alfabetizados (además, el 50 por 100 de los analfabetos falló en todas las pruebas). De aquí concluían los autores del trabajo que la capacidad de manipular fonos de forma consciente no se obtiene automáti- camente a una edad, sino que más bien parece depender del aprendizaje.

CUADRO III

Resultados de Morais y otros (1979)

\begin{tabular}{lcccc}
\hline & \multicolumn{2}{c}{ Adición } & \multicolumn{2}{c}{ Sustracción } \\
\hline & Palabras & Pseudopalabras & Palabras & Pseudopalabras \\
Analfabetos & $46 \%$ & $19 \%$ & $26 \%$ & $19 \%$ \\
Alfabetizados & $91 \%$ & $71 \%$ & $.87 \%$ & $.73 \%$ \\
\hline
\end{tabular}

Read y colaboradores suyos realizaron el mismo tipo de prueba a dos grupos de adultos que habían asistido a la escuela diez años aproximadamente 6 . Un grupo había aprendido a leer el pin-yin (método alfabético introducido por Mao en 1949), y el otro, los logogramas chinos habituales. Los resultados que se obtuvieron ponían una-vez más de manifiesto que sólo mostraban capacidad de análisis fonético quienes habían aprendido a leer en un alfabeto (el pin-yin).

Estos trabajos (y otros muchos que, por razón de brevedad, no aparecen aquí comentados) tienen una conclusión común: que la capacidad para realizar tareas de análisis fonético no se debe a la maduración a una determinada edad, sino que parece ser fruto de un aprendizaje y ejercitación específicos que el niño realiza cuando se le pone ante actividades que las requieren. 


\section{Ejercicios de entrenamiento de análisis fonético}

Si tal como he argumentado, el análisis fonético de la palabra es una actividad ligada al aprendizaje de la lectura en un sistema alfabético, es preciso preguntarse cuáles serían los ejercicios más apropiados para facilitar este aprendizaje. Algunos autores ${ }^{7}$ proponen ejercicios y procedimientos en este sentido; todos ellos se reducen a añadir y sustraer fonos en palabras, segmentar palabras y sílabas, buscar fonemas comunes en palabras, etc. Otras estrategias sugieren el interés de utilizar apoyos visuales como diagramas o dibujos que representan segmentos silábicos o fonéticos ${ }^{8}$; aunque, según $A$. Content, éstos no parecen tener más utilidad que la de facilitar una comprensión de la tarea misma que se les pide realizar.

En cualquier caso, todos estos ejercicios no son más que indicativos; las estrategias particulares pueden ser creadas por el propio maestro para su situación particular, o podémos encontrar ejemplos interesantes en algunos métodos de lectura 9 . Lo realmente interesante está en ir descubriendo los procesos implicados en el hecho de leer.

\section{Notas}

1 Un planteamiento interesante es el modelo que expone Alegría (1985) avalado por un buen número de trabajos experimentales: Coltheart (1978), Baron y Treiman (1980) y Jorn y Share (1983).

2 El grafema corresponde en la escritura alfabética a una letra determinada, ya sea mayúscula, minúscula, cursiva, etc. Es, por tanto; la mínima unidad de escritura, no susceptible de ser dividida. El fonema es la más pequeña unidad fonológica de una lengua; es una unidad abstracta. El sonido es la unidad fonética, la unidad mínima del habla. Se refiere a la realización concreta de los fonemas. Así, el número de fonemas es fijo y limitado, en una lengua, pero no el de sonidos. La paläbra «zona» tiene cuatro fonemas que, en el habla, varían según la producción particular de los sujetos. En este trabajo utilizamos el término fono como equivalente a sonido, por ser más usual en la bibliografía que hemos consultado.

3 En mi opinión, algunos problemas de la enseñanza de la lectura se derivan de la falta de estudios básicos en psicología de la lectura, lo que repercute, sin duda, en una fundamentación débil, en unos casos, e incluso errónea en otros a la hora de elegir o crear métodos concretos para enseñar a leer.

4 La experiencia consiste en hacer mirar al sujeto-una pantalla donde aparece una secuencia de letras durante un tiempo breve (las letras pueden formar palabras como, por ejemplo, «brain», o pseudopalabras, como «slint»); este tiene que decir rápidamente si se trata de una palabra o no. El resultado que interesa observar aquí es que la respuesta «no» a pseudopalabras homófonas de palabras es más lenta que la respuesta «no» a pseudopalabras no homófonas, lo que implica que la decisión es/no es tomada teniendo en cuenta la versión fonológica de la secuencia de letras presentada.

5 Actualmente subsiste algún silabario, como es el caso del japonés.

6 Citado por Alegría (1985).

7 Alain Content (1985) cita un buen número de programas de ejercicios en este sentido: En U.S.A. se practicaron, por ejemplo, los de Williams (1977); Wallach y Wallach (1976) y Rosner (1975), todos ellos relativos a análisis y/o fusión de fonos, pensados como plan pedagógico para niños retardados en lectura. En la Unión Soviética, Elkonin desarrolló un método como etapa previa a la enseñanza de la lectura que se adoptó oficialmente. El autor lo basa en el análisis de la palabra fundamentalmente oral, acentuando los segmentos y haciendo pausas entre ellos. Lewkovicz (1980) propone dos principios para aprender a segmentar, pronunciar la palabra muy lentamente y, en segundo lugar, poner atención sobre los índices articulatorios.

8 Content (1985) cita algunos ejemplos de estos trabajos, Marsh y Mineo (1977), Lewkovics y Low (1979). He visto ejemplos en el método 27 frases para enseñanza a leer, de Inizan, Madrid, Pablo del Río, 1980.

9 Pensamos en ejercicios que pueden considerarse como juegos lingüísticos divertidos, como «veo-veo», el tren de palabras (decir palabras que empiezan por la última sílaba o «fonema» con que termina la que ha dicho el anterior), elegir palabras que empiezan por la misma. letra (fonema), etc. En el método citado de Inizan hay un conjunto de juegos fonológicos muy interesantes. Asimismo, en el método Letra por letra. Bases para la didáctica de la lectura y la escritura, de Marta Mata y otros, Barcelona, La Galera, 1979. 


\section{Resumen}

En este artículo se apuntan algunas lineas de reflexión sobre qué babilidades son puestas en juego al adquirir la capacidad de leer en los sistemas alfabéticos. Previamente se resume la evolución de los sistemas de escritura a fin de señalar las particularidades del sistema alfabeto frente al silábico y alfabético. Se revisan finalmente algunos trabajos que dan cuenta del papel que tiene el análisis de la palabra y más específicamente el análisis fonético en el aprendizaje de la lectura, cuándo y cómo se adquiere esta babilidad.

\section{Summary}

This article points out some reflection lines about the skills that play a role in the acquirement of capability to read in the alphabetical systems. First of all, we summarize the evolution of writing systems in order to remarck particularities of alphabetical systems as compared to syllabic and logographic: Finally, some studies about the role that word analysis are reviewed and more specifically phonetic analysis, plays in the learning to read and when and bow this skill is reached.

\section{Résumé}

Dans cet article on signale quelques lignes de reflexion sur les aptitudes qu'il faut mettre en jeu pour acquérir la capacité de lire dans les systèmes alphabétiques. On fait, préalablement, un résumé sur l'évolution des differents systèmes d'écriture à fin de remarquer les particularités du système alphabétique par rapport aux systèmes syllabique et logographique. Finalement on fait une révision de certains articles que permettent mettre en évidence l'importance de l'analyse de la parole, ou plus specifiquement l'analyse phonétique, sur l'apprentissage de la lecture, ainsi que quand et comment on acquis cette capacité.

\section{Referencias}

ALEgrfa, J. y MóraIS, J.: «Le développement de l'habilité d'analyse phonétique de la parole et l'apprentissage de la lecture». Archives de psychologie, 1979, 47, 251.270

Alegrfa, J. Pignot, E. t MORAIS, J.: «Phonetic analysis of speech and memory codes in beginning readers». Memory and Cognition, 1982, 10, 451-456.

ALEGRfA, J.: «Por unenfoque psicolingüístico del aprendizaje de la lectura y sus dificultades». Infancia y Aprendizaje, 1985, 29, 79-94.

BARON, J. y TREIMAN; R.: «Use of orthography in reading and learning to read». Kavanach, J. F. y Venezky, R. L. (eds.), Orthography reading and dyslexia. Baltimore, Univ. Park Press, 1980.

BRADLEY, L. y BRYANT, P. E.: «Difficulties in auditory organization as a possible cause of reading backwardness». Nature, 271, 1978, 746-747.

ColtheART, M.: «Lexical access in simple reading tasks». Underwood, G. (ed.), Strategies of information processing, London Academic Press, 1978.

CONTENT, A.: «L'analyse phonétique explicite de la parole et l'acquisition de la lecture». L'Année Psychologique, $1984,84,555-572$.

- «Le dévéloppement de l'habilité d'analyse phonétique de la parole». L'Année Psychologique, 1985, 85, 73-99.

Ferreiro, E. y Teberosky, A.: Los sistemas de escritura en el desarrollo del niño. México, Siglo XXI, 1979.

Fox, B. y RouTH, D. K.: «Phonetic analysis and severe reading disability in children». Journal of Psycholin. guistic Research, 1980, 9, 115-119.

- "Reading disability, phonemic analysis and dysphonetic spelling: a follow-up-study». Journal of Clinical Child Psychology, 1983, 12, 28-32.

GelB, I. J.: Historia de la escritura. Madrid, Alianza, 1976.

Jorm, A. F. y Share, D. L.: «Phonological recoding and reading acquisition». Applied Psycholinguistics, 1984, 4, $103-147$.

LAZARo Carreter, F.: Diccionario de términos filológicos. Madrid, Gredos, 1971.

Liberman, I. Y. y otros: «Perception of the speech code». Psychological Review, 1967, 74, 431-461.

- «Reading and awereness of linguistic segments». Journal of Experimental Child-Psychology, 18, 1974, 201-212.

Morais, J., Cary, L., Alegria, J. y Bertelson, P.: «Does awarencess of speech as a sequence of phones arise spontaneously? Cognition, 1979, 7, 323-331.

Morais, J., Cluytens, M., Alegria, J.: «Segmentation abilities of dyslexics and normal readers». Perceptual and Motor Skill, 1984, 58, 221-222.

ROSNER, J. y SIMON, D. P.: «The auditory analysis test: an initial report». Journal of learning Disabilities, 1971 , 4, 384-392.

RubENSTEIN, H., Lewis, S. y RubEnStein, M. A.: «Evidence for phonemic recoding in visual word recognition». Journal of Verbal Learning and Verbal Behavior, 10, 1971, 645-657.

Zhurova, L. Y.: "The development of analysis of words into their sounds by preschool children», Ferguson, C. A. y Slobin, D. I. (eds.), Studies of child language development. Nueva York, Holt, Rinehart y Winston 1973 . 